\title{
Femmes et fonction publique
}

Un risque calculé de déclassement?

Women and Public Sector: Is there a Calculated Risk of Overeducation?

\section{Vanessa Di Paola et Stéphanie Moullet}

\section{CpenEdition}

\section{Journals}

Édition électronique

URL : http://journals.openedition.org/travailemploi/1655

DOI : 10.4000/travailemploi. 1655

ISSN : 1775-416X

Éditeur

DARES - Ministère du Travail

Édition imprimée

Date de publication : 15 décembre 2009

Pagination : 47-61

ISSN : 0224-4365

Référence électronique

Vanessa Di Paola et Stéphanie Moullet, «Femmes et fonction publique », Travail et Emploi [En ligne], 120 | octobre-décembre 2009, mis en ligne le 31 janvier 2011, consulté le 30 avril 2019. URL : http:// journals.openedition.org/travailemploi/1655; DOI : 10.4000/travailemploi.1655 


\title{
Femmes et fonction publique: un risque calculé de déclassement?
}

\author{
Vanessa di Paola (*), Stéphanie Moullet (*)
}

Parce que la fonction publique est attractive, davantage encore pour les femmes que pour les hommes, les jeunes pourraient faire des choix éducatifs les conduisant à se former au-delà du niveau normalement requis pour faciliter leur insertion dans ce secteur. Cet article s'intéresse au déclassement des jeunes femmes comparativement à celui des jeunes hommes, dans la fonction publique, relativement au secteur privé. À l'approche usuelle en terme de niveau de formation, s'ajoute celle en terme de rémunération, les jeunes femmes pouvant transiger sur leur salaire plus que les jeunes hommes. Il s'agit de savoir si l'attrait des jeunes femmes pour la fonction publique les conduit à relativiser une situation objective de déclassement moins favorable que celle des jeunes hommes? Les données utilisées sont issues de l'enquête "Génération 98》 (CÉREQ) qui interroge en 2001 les jeunes sortis de formation initiale trois ans plus tôt. On montre que les femmes en emploi dans la fonction publique ont, au regard du salaire comme du niveau de formation, un consentement à payer - le fait d'avoir un sentiment de déclassement plus faible, à déclassement objectif donné - plus important que celui des hommes (quel que soit leur secteur d'emploi), mais aussi que celui des femmes en emploi dans le secteur privé. L'accès à un emploi à vie accroît ce consentement à payer, c'est-à-dire conduit à minimiser davantage encore, le décalage éventuel entre niveau de formation ou de salaire et ressenti.

La détérioration des conditions d'insertion au cours des dernières décennies fait du choix du secteur d'emploi, au-delà de ses déterminants en termes de préférences individuelles, une question centrale dans l'analyse des débuts de vie active. On sait notamment que le taux de candidature à la fonction publique (le nombre d'inscrits rapporté au nombre de postes offerts) croît avec le taux de chômage, et ce en particulier au sein de la population féminine (Fougère, Pouget, 2003). Les travaux portant sur la dimension sexuée de l'insertion des jeunes laissent aussi à penser que l'interaction entre le contexte du marché du travail et les goûts individuels conduisent les hommes et les femmes à se positionner différemment sur les secteurs public et privé. En particulier dans la fonction publique, on a mis en évidence (Di Paola, Moullet, 2003) que les trajectoires d'insertion se distinguent essentiellement selon le sexe: les jeunes femmes accèdent plus rapidement que les jeunes hommes après leur sortie de formation initiale au statut de fonctionnaire.

Plus largement, l'accès rapide à la fonction publique après la fin des études peut traduire, de la part des femmes, une démarche rationnelle: parce que ce secteur présente pour elles une assurance

(*)LEST-CNRS ; di-paola@univmed.fr ; stephanie.moullet@ univ-med.fr contre les aléas du marché privé (conditions de travail meilleures, moindre discrimination à l'embauche, discrimination salariale limitée, choix de métiers...), elles feraient des choix éducatifs en terme de spécialité et de niveau de formation leur facilitant l'entrée dans la fonction publique. En particulier, l'aménagement du temps de travail en réduisant les coûts de garde des enfants, facilite leurs modalités de conciliations entre vie familiale et vie professionnelle et rationalise pour les femmes le choix de ce secteur d'emploi (Odena, 2005; GARNER et al., 2005).

Le déclassement, usuellement défini comme un décalage entre le niveau de formation initiale acquis et le niveau normalement requis (pour passer les concours d'accès à la fonction publique par exemple) peut alors être envisagée et étudiée comme une lecture des choix des jeunes femmes. Le souhait d'accéder à la fonction publique pourrait en effet se traduire par un «consentement à payer» mesurable en terme de déclassement à l'embauche : il s'agit de se former au-delà du niveau normalement requis pour augmenter les chances de devenir fonctionnaire sans exprimer d'insatisfaction relative au décalage qui en découle. Ce décalage pourrait pourtant se révéler important pour les jeunes femmes fonctionnaires. Cependant, la manière dont ce désajustement est mesuré peut nuancer cette hypothèse. En effet, du fait de sa définition basée sur les comportements les plus fréquents, le déclasse- 
ment statistique sera faible pour les jeunes femmes fonctionnaires si elles sont nombreuses à faire le choix de se surdiplômer (Di PAOLA, et al., 2005). De même, leur sentiment d'un désajustement entre leur niveau de diplôme et le niveau de l'emploi occupé, même s'il est effectif, parce qu'anticipé et accepté, peut s'avérer moins important pour elles que pour leurs homologues masculins dans une situation de désajustement similaire. À côté de l'analyse des désajustements sur la base des niveaux de formation, se pencher sur les désajustements en matière de rémunération peut éclairer les différenciations des comportements des femmes et des hommes. En effet, si la littérature a montré qu'hommes et femmes n'attachent pas la même importance à la rémunération dans la satisfaction qu'ils tirent de leur activité professionnelle (BAUDELOT et al., 2003), on peut se demander comment le sentiment à l'égard d'un désajustement de rémunération effectif s'exprimera pour les femmes relativement aux hommes. Est ce que, à situation objective donnée, les femmes se sentent moins déclassées que les hommes en termes de rémunération et comment le fait d'être en emploi à vie (fonctionnaire) intervient-il pour les uns et les autres sur ce sentiment?

Que ce soit en termes de salaire ou en termes de niveau de formation initiale, le fait, pour les femmes, d'avoir éventuellement, un sentiment de déclassement plus faible que les hommes, à déclassement objectif donné, traduit ce que l'on appellera leur «consentement à payer» pour être en emploi dans la fonction publique. La notion de consentement à payer est envisagée ici dans un contexte de risque, inspirée des travaux d'économie de la santé (1). Un décalage entre mesure objective et subjective plus importante pour les femmes (minimisation en termes de ressenti de la situation objective) qui font face à des risques plus grands que les hommes sur le marché du travail sera lu comme un consentement à payer des femmes.

Dans un premier temps, l'objet de notre contribution est de conduire une analyse descriptive des déclassements des jeunes entrant dans la vie active. L'analyse se veut comparative sur deux aspects: celui du secteur et celui du genre. Ainsi nous étudierons la situation des jeunes femmes employées dans la fonction publique (et notamment celles fonctionnaires) comparativement à celles en emploi dans le secteur privé; mais aussi celle des jeunes hommes employés dans la fonction publique. Un effort particulier consistera à appréhender l'ampleur du déclassement en recourant à différentes mesures du phénomène.

(1) Il ne s'agit pas ici d'évaluer le consentement à payer des jeunes (le prix maximum que les jeunes sont prêts à payer pour accéder à l'emploi) mais d'emprunter modestement cette notion pour lire le déclassement comme un consentement, c'est-à-dire le résultat d'arbitrage en termes de risque (à rapprocher de la méthode des préférences révélées).
Dans un second temps de l'analyse, on se propose d'étudier les déterminants individuels du déclassement vu comme un consentement à payer. Une telle analyse suppose de considérer les déterminants de la participation au marché du travail ainsi que ceux du choix du secteur d'emploi. En effet, l'individu qui est en emploi peut être déclassé alors que celui qui n'est pas en emploi a peut-être refusé de se déclasser. Il conviendra donc de tenir compte dans la modélisation retenue du fait que l'accès à l'emploi (plutôt que l'absence de participation au marché du travail ou le chômage) n'est pas indépendant du consentement individuel à se déclasser. Ceci étant contrôlé, nous chercherons à évaluer dans quelle mesure être en emploi dans la fonction publique d'une part, être fonctionnaire d'autre part est explicatif d'un sentiment de déclassement à l'embauche, pour les femmes et les hommes comparativement. Ainsi, étant donné la situation de déclassement objectif, les femmes se sentent-elles moins déclassées que les hommes? Travailler dans la fonction publique plutôt que dans le secteur privé diminue-t-il le ressenti de déclassement? Enfin, l'accès au statut de fonctionnaire réduit-il encore ce ressenti? Les résultats diffèrent-ils selon le sexe? Les réponses à ces questions pourront être lues comme des consentements à payer dans la mesure où la situation de déclassement objective est contrôlée.

\section{Les données}

Les données utilisées sont issues de l'enquête «Génération 98» réalisée par le Centre d'études et de recherches sur les qualifications (CÉREQ). Elles concernent 55000 sortants du système éducatif en 1998 tous niveaux de formation et toutes spécialités de formation confondues parmi les 750000 primo-sortants à cette date. Les données sont rétrospectives. Elles permettent d'analyser les trois premières années de vie active au regard de la formation initiale. Elles autorisent donc la comparaison des modalités d'insertion professionnelles des jeunes selon leur niveau d'éducation dans une même conjoncture économique. L'enquête a pour objet de rendre compte des différentes composantes des parcours d'insertion professionnelle: elle fournit des informations individuelles, sociodémographiques et relatives au parcours scolaire ainsi qu'aux différentes séquences d'emploi ou de non-emploi. Une séquence étant définie comme une durée passée dans un état, une séquence d'emploi correspond à une période d'emploi exercée au sein d'un même établissement sans interruption.

Pour chaque séquence d'emploi, le secteur d'activité, la profession et catégorie socioprofessionnelle Profession et Catégorie socioprofessionnelle (PCS) occupée, le contrat et le temps de travail ainsi que le salaire mensuel perçu, primes incluses, sont connus. 
Tableau 1 : Statistiques descriptives relatives aux personnes en emploi à la date de l'enquête et dans la fonction publique (FP) en \%

\begin{tabular}{|c|c|c|c|c|c|c|c|}
\hline \multirow{2}{*}{ Caractéristiques } & \multicolumn{2}{|c|}{ Ensemble } & \multicolumn{2}{|c|}{ Hommes } & \multicolumn{2}{|c|}{ Femmes } & \multirow{2}{*}{$\begin{array}{l}\text { Effectifs ensemble de la } \\
\text { population en emploi }\end{array}$} \\
\hline & & FP & & FP & & FP & \\
\hline Hommes & $53^{*}$ & $40 *$ & - & - & - & - & 314000 \\
\hline Niveau I (1) & 9 & 9 & 10 & 10 & 9 & 8 & 54000 \\
\hline Niveau II & 11 & 22 & 8 & 18 & 15 & 25 & 67000 \\
\hline Niveau III & 21 & 23 & 18 & 18 & 24 & 26 & 124000 \\
\hline Niveau IV & 18 & 14 & 17 & 15 & 17 & 14 & 102000 \\
\hline Niveau IV+ & 12 & 15 & 11 & 18 & 13 & 14 & 70000 \\
\hline Niveau V & 24 & 14 & 29 & 17 & 18 & 11 & 139000 \\
\hline Niveau Vb & 3 & 2 & 4 & 3 & 2 & 1 & 20000 \\
\hline Niveau VI & 2 & 1 & 3 & 1 & 2 & 1 & 13000 \\
\hline Secteur priv & 77 & - & 83 & - & 71 & - & 456000 \\
\hline Fonction publique, dont : & 23 & 100 & 17 & 100 & 29 & 100 & 133000 \\
\hline Education nationale & 6 & 26 & 3 & 18 & 9 & 31 & 34000 \\
\hline Collectivités territoriales & 4 & 16 & 3 & 16 & 4 & 15 & 21000 \\
\hline Hôpitaux & 3 & 15 & 1 & 7 & 6 & 21 & 21000 \\
\hline Autres dans la FP d'Etat & 10 & 43 & 10 & 59 & 10 & 33 & 57000 \\
\hline Fonctionnaires & 6 & 25 & 4 & 26 & 7 & 25 & 34000 \\
\hline Cdi & 59 & - & 63 & - & 55 & - & 348000 \\
\hline \multicolumn{8}{|l|}{ PCS : } \\
\hline cadre prof supérieure & 13 & 17 & 14 & 10 & 12 & 13 & 78000 \\
\hline prof intermédiaire & 29 & 42 & 25 & 17 & 33 & 38 & 168000 \\
\hline employée & 29 & 33 & 16 & 17 & 44 & 27 & 172000 \\
\hline ouvrier & 29 & 8 & 45 & 9 & 11 & 2 & 171000 \\
\hline Total & 589000 & 133000 & 313000 & 53000 & 276000 & 80000 & 589000 \\
\hline
\end{tabular}

(1) On trouvera en annexe la nomenclature des niveaux de formation.

Source: enquête «Génération 98 », première interrogation, CEREQ.

Champ: ensemble des jeunes sortis en 1998 de formation initiale en emploi en 2001, effectifs pondérés.

${ }^{(*)}$ Note de lecture: $53 \%$ des jeunes sortis du système éducatif en 1998 en emploi en 2001 sont des hommes et $40 \%$ de ceux qui sont en emploi dans la fonction publique à cette date sont des hommes.

La variable décrivant le secteur public ou privé de l'emploi occupé est également disponible et relève d'une déclaration individuelle. À partir de cette dernière et sur la base du nom déclaré de l'employeur, il devient possible de distinguer les trois fonctions publiques - fonction publique d'État, territoriale et hospitalière - , et au sein de la fonction publique d'État, de séparer les types d'employeur, Éducation nationale, Armée et autres ministères. Les entreprises publiques nationalisées sont également identifiables mais ne font pas partie de la fonction publique.
Dans cette analyse, nous retenons l'ensemble des jeunes qui occupent un emploi en mars 2001, à savoir trois ans après la sortie de la formation initiale (2). Cela correspond à une population de près de 45000 individus soit $82 \%$ de la population totale. On observe (voir tableau 1) que la population

(2) Le service national, encore en vigueur pour les jeunes hommes de la Génération 98, a pu différer leur entrée sur le marché du travail. Pour autant, en 2001, tous les jeunes hommes de l'enquête sont dégagés de ces obligations (CEREQ, 2002). 
comporte $23 \%$ de jeunes en emploi dans la fonction publique au moment de l'enquête, avec une part des jeunes femmes très importante puisque $29 \%$ d'entre elles sont en emploi dans la fonction publique, contre seulement $17 \%$ des jeunes hommes. De plus, en terme de stabilité de l'emploi, on constate que $6 \%$ des jeunes sont fonctionnaires et $59 \%$ sont en CDI à la date d'enquête. La répartition entre les différentes catégories sociales met en avant une distribution différente au sein de la fonction publique: la part des ouvriers n'y est que de $8 \%$ (contre $29 \%$ par ailleurs), celle des professions intermédiaires et des cadres y est de $59 \%$ contre $42 \%$ dans le secteur privé. Les niveaux de formation, conformément aux répartitions entre catégories sociales, sont plus élevés au sein de la fonction publique: les niveaux II et III de formation ( $\mathrm{du}$ bac +2 au bac + 4) représentent près de $45 \%$ des jeunes dans la fonction publique, contre seulement environ $30 \%$ pour l'ensemble des jeunes. L'écart de formation entre les jeunes hommes et les jeunes femmes est de même ampleur que l'on considère l'ensemble de la population ou seulement les jeunes de la fonction publique: les jeunes femmes sont plus diplômées que les jeunes hommes.

\section{Déclassement, genre et secteur d'emploi}

Mesurer le déclassement suppose de définir la population des déclassés. Usuellement, trois définitions sont retenues: l'approche subjective, l'approche statistique et l'approche normative. Le concept usuel de déclassement renvoie à un décalage entre le niveau de formation et le niveau de qualification de l'emploi. Une autre manière de l'envisager est de le penser en termes de rémunération. On peut alors définir un déclassement de salaire reposant sur l'existence d'un écart entre ce qui perçu et ce qui devrait «normalement» l'être du point de vue statistique ou subjectif.

\section{Mesurer le déclassement: quels indicateurs retenir?}

Pour mesurer le déclassement subjectif, on choisit de se référer directement à l'auto-appréciation que font les jeunes de leur situation. L'enquête permet de mesurer le déclassement subjectif de niveau à travers la réponse à la question: « $\dot{A}$ propos de cet emploi, diriez-vous que vous étiez utilisé: (A) à votre niveau de compétences, $(B)$ en dessous de votre niveau de compétences, (C) au-dessus de votre niveau de compétences.». Ainsi, se dégage une mesure du déclassement subjectif codée 1 si le jeune a le sentiment d'être employé en dessous de son niveau de compétences et 0 sinon. Notons qu'ici, contrairement aux approches qui vont suivre, on se réfère aux compétences du jeune et non pas

\section{Encadré 1}

\section{La mesure du déclassement de niveau}

Notons $\mathrm{X}$, le niveau de diplôme et $\mathrm{Y}$ la catégorie socioprofessionnelle, pouvant prendre respectivement les valeurs $X_{1}, \ldots, X_{i}, \ldots, X_{p}$ et $Y_{1}, \ldots, Y_{i}, \ldots . Y_{q}$. Nous avons retenu ici les niveaux de diplôme suivants: non qualifiés, CAP ou BEP, bac, bac $+2,2^{\mathrm{e}}$ cycle et $3^{e}$ cycle de l'enseignement supérieur; les catégories socioprofessionnelles sont au nombre de sept: ouvriers non qualifiés, employés non qualifiés, ouvriers qualifiés, employés qualifiés, techniciens, professions intermédiaires et cadres.

Notons, d'une part, que l'indicateur de déclassement est sensible à l'éclatement retenu de ces deux variables et d'autre part que la catégorie des cadres englobe une grande hétérogénéité des diplômes du supérieur, allant de la licence au doctorat et grandes écoles, limitant ainsi l'évaluation des situations de déclassement des plus diplômés.

Soient $\mathrm{n}$ l'effectif total, nij l'effectif du couple $\left(X_{i}, Y_{j}\right), n_{i}$. et $n_{\cdot j}$ les effectifs marginaux des modalités $X_{i}$ et $Y_{j}$. Sous l'hypothèse d'indépendance entre $X$ et $Y$, l'effectif théorique du couple $\left(X_{i}, Y_{j}\right)$ est $n^{*}{ }_{i j}=\left(n_{i} n_{j}\right) / n$. Pour chaque couple $(i, j)$, on peut alors considérer que la situation diplôme - catégorie socioprofessionnelle est:

«normale» si $n_{i j}>n^{*}{ }_{i j}$ puisqu'il s'agit d'une concordance fréquente entre les modalités $X_{i}$ et $Y_{j}$;

«atypique» si $n_{i j}<n^{*}{ }_{i j}$ car la concordance est rare entre les modalités $X_{i}$ et $Y_{j}$. Dans ce deuxième cas, on détermine si la situation "atypique " correspond à du "sur classement " ou à du "déclassement", en situant "hiérarchiquement" le couple (i,j) par rapport aux couples $\left(i, j^{\prime}\right)$ et $\left(i^{\prime}, j\right)$ considérées comme «normales».

seulement aux diplômes détenus. Toutefois, le déclassement étant mesuré ici trois ans seulement après la sortie du système éducatif, les compétences sont encore largement déterminées par la formation initiale et donc le diplôme obtenu. De la même manière, le déclassement subjectif de salaire repose sur la réponse à la question suivante: $« P a r$ rapport au travail que vous faites, diriez-vous que vous êtes: (A) très bien payé, (B) plutôt bien payé, (C) plutôt mal payé et (D) très mal payé». Ces deux derniers items sont regroupés pour définir cet indicateur: si le jeune déclare être plutôt mal payé ou très mal payé, la variable de déclassement subjectif salarial prend la valeur 1 , et 0 sinon.

Les approches subjectives permettent de s'affranchir des difficultés de définition de nomenclatures (Di Paola, et al., 2005). Pour autant, elles ne sont pas sans avoir de limites (GIREt, 2005). En particulier, l'indicateur subjectif de déclassement fondé sur la notion de compétence n'est pas nécessairement interprété comme un désajustement entre niveau de l'emploi et niveau de formation initiale. En effet, la subjectivité individuelle peut conduire à une confusion des dimensions liées au poste 
Tableau 2: Table de correspondance statistique PCS-diplôme : déclassement statistique ou objectif de niveau

\begin{tabular}{|l|c|c|c|c|c|c|c|}
\hline & ONQ & ENQ & OQ & EQ & Tech & PI & Cadres \\
\hline Non qualifiés & Norme & Norme & Norme & Norme & Norme & Norme & Norme $^{(*)}$ \\
\hline CAP ou BEP & Norme & Norme & Norme & Norme & Norme & Norme & Norme \\
\hline BAC & Déclassé & Norme & Norme & Norme & Norme & Norme & Norme \\
\hline Bac $+\mathbf{2}$ & Déclassé & Déclassé & Déclassé & Norme & Norme & Norme & Norme \\
\hline $\mathbf{2}^{\mathbf{e}}$ cycle & Déclassé & Déclassé & Déclassé & Norme & Norme & Norme & Norme \\
\hline $\mathbf{3}^{\mathbf{e}}$ cycle & Déclassé & Déclassé & Déclassé & Déclassé & Déclassé & Déclassé & Norme \\
\hline
\end{tabular}

$\left({ }^{*}\right)$ Les jeunes surclassés sont considérés ici comme étant dans la situation «normale».

Source : enquête « Génération 98 », première interrogation, CÉREQ.

Champ : ensemble des jeunes sortis en 1998 de formation initiale en emploi en 2001.

occupé: outre l'adéquation au niveau de formation initiale, peuvent se mêler les aspects de satisfaction dans l'emploi et de salaire. D'une manière générale, les jugements subjectifs, notamment l'appréciation par les individus de leur salaire, sont largement dépendants des caractéristiques de l'emploi et des conditions de travail ainsi que des caractéristiques, en particulier le sexe, de ceux qui les expriment (Godechot, Gurgand, 2000).

La mesure statistique du déclassement de niveau considère que le niveau «normalement» requis pour occuper un poste peut être défini par rapport au niveau de diplôme du plus grand nombre de personnes occupant ce type d'emploi. Cette mesure, que l'on qualifie d'objective par opposition au déclassement subjectif, s'appuie sur les tableaux de contingence croisant diplômes et catégories socioprofessionnelles. Elle est définie par une analyse des écarts à l'indépendance (FORGEOT, Gautié, 1997) (voir encadré 1).

Le niveau de déclassement statistique ou objectif de niveau est codé «1» si le jeune est identifié comme déclassé, et il prend la valeur « 0 » s'il est dans la norme ou s'il est surclassé. La table de correspondance est reportée, elle est construite pour l'ensemble de la population active occupée, quel que soit le secteur d'emploi. Le secteur privé et les trois fonctions publiques renvoient à des structures d'emploi différentes (davantage d'emploi de techniciens dans le secteur privé par exemple). La mesure statistique du déclassement étant fondée sur ces structures d'emploi, l'indicateur statistique du déclassement n'aurait pas été le même s'il avait été construit séparément pour chaque secteur d'emploi. Notre choix consiste à caractériser la situation de chaque individu en référence à l'ensemble de la population en emploi et non pas de manière restrictive à la population de son secteur d'emploi.

$\mathrm{Du}$ point de vue salarial, on suppose qu'un individu dont la rémunération est inférieure à celle de $50 \%$ des individus de niveau de diplôme immédiatement inférieur est déclassé statistiquement ou objectivement du point de vue du salaire (NAUZEFichet, Tomasini 2001, Cahuzac, Di Paola, 2005). Le recours à cette définition suppose que la hiérarchie des diplômes suit celle des salaires, ce qui est vérifié sur la population des jeunes actifs trois ans après la fin de leurs études. Ainsi, un jeune dit déclassé statistiquement ou objectivement du point de vue du salaire aurait la même rémunération avec le niveau de diplôme inférieur au sien: pour cette approche, le déclassement correspond donc à une moindre rentabilisation de la formation initiale. Le choix de la médiane comme seuil dans la définition du déclassement salarial a une part d'arbitraire, d'autres seuils auraient produit des résultats différents. Pour autant, la médiane constitue le seuil le plus intelligible et celui retenu dans la plupart des travaux. Cet indicateur sera dans la suite de l'article qualifié d'objectif par opposition au déclassement salarial subjectif, bien qu'il dépende du choix d'un seuil.

La mesure normative du déclassement repose sur une approche adéquationniste faisant correspondre des niveaux de formation avec une nomenclature des emplois sur la base d'analyse de contenus d'emploi et de formation conduites par des experts. C'est la voie ouverte par AfFichard (1980) dans le secteur privé uniquement. Pour le secteur privé comme pour la fonction publique, le déclassement normatif relève d'une objectivation externe des situations d'emploi. Cette mesure est certainement la plus intuitive, et en particulier dans la fonction publique caractérisée par des niveaux précis de recrutement par concours, elle pourrait permettre une réflexion sur l'écart entre norme juridique d'inscription aux concours de la fonction publique et la réalité des diplômes possédés par les candidats, dont on sait qu'ils sont supérieurs (Fougère, Pouget, 2003). Une telle mesure du déclassement est cependant délicate, puisqu'elle suppose soit de se référer aux normes définies par AFFICHARD (1980) qui sont aujourd'hui anciennes et qui ne considèrent pas la fonction publique; soit de retenir les niveaux requis pour passer les concours, donnant alors lieu à plusieurs difficultés. La première est liée à l'éclatement des niveaux de concours spécifiques à certains métiers, la seconde est celle du choix de la norme à retenir pour les non-fonctionnaires de la fonction publique qui ne sont pas soumis au recrutement par concours. Dans un précédent article, Di PaOla et al. (2005) ont montré, en définissant le déclassement 
normatif à partir d'une norme légale simplifiée (les diplômes exigés pour passer les concours d'entrée dans la fonction publique, y compris pour les non titulaires), le caractère absorbant d'une telle norme: tous les individus déclassés du point de vue statistique, le sont systématiquement du point de vue normatif. Cette particularité est probablement liée à une définition trop vaste de la norme, mais actuellement délicate à dépasser.

Ainsi, l'analyse du déclassement normatif renvoie à une interrogation sur les niveaux de concours; la définition statistique quant à elle met en évidence le rapport à une norme effective cette fois et non plus légale, c'est la distribution des diplômes au sein de chaque catégorie qui est considérée. Cela renvoie dans les deux cas aux pratiques en vigueur sur ce marché du travail de l'emploi public et interroge de fait les stratégies de l'employeur ainsi que celle de l'individu: dans quelle mesure l'employeur public subit ou exploite le fait que les personnes recrutées ont un niveau d'étude supérieur au niveau requis légalement? Enfin, la mesure subjective permet d'appréhender le positionnement de l'individu quant à l'utilisation de ses propres compétences dans son emploi, et l'on peut envisager que ce sentiment est influencé parce que l'individu observe au sein de son groupe de référence.

Ici, seules les approches objectives et subjectives sont retenues dans la mesure où, contrairement à la mesure normative, elles tiennent compte de la situation de chaque individu relativement aux situations des autres. Être objectivement déclassé signifie, en effet, ne pas être dans la situation que connaît la majorité de la génération de jeunes considérés (de même niveau de formation initiale). De manière semblable, le sentiment de déclassement de chacun des jeunes peut être lu comme étant construit en référence à son entourage (phénomène dit des préférences adaptatives, SEN 1992), par rapport à un niveau jugé moyen dans l'entreprise dans laquelle il est employé, ou bien par rapport à un niveau moyen dans un sous-groupe de référence (groupe d'amis, famille, etc.). Plus globalement, ce ressenti dépend des caractéristiques de chacun, de sa trajectoire scolaire et sur le marché du travail, de sa propre projection personnelle et professionnelle, de sa mobilité sociale ...

\section{Déclassement objectif et subjectif: des différences sexuées selon le secteur d'emploi}

Chacun des indicateurs présentés ci-dessus est calculé relativement à la situation au moment de l'embauche de l'emploi occupé à la date d'enquête, mars 2001.

Les premiers résultats (voir tableau 3) font en premier lieu apparaitre un faible taux de jeunes déclassés du point de vue objectif au regard de leur diplôme: moins de $10 \%$ des jeunes en 2001 occupent un emploi inférieur à leur niveau de formation initiale. Avec un indicateur de même nature statistique mais où la norme statistique est construite en référence à des cohortes plus anciennes de dix années (NAUZE, Fichet, TomasinI, 2005), près de $24 \%$ de ces mêmes jeunes sont identifiés comme déclassés alors que leur situation n'a pas changé. Ces différences renvoient à la nature relative de la définition de l'indicateur objectif de déclassement. Il est en effet très sensible d'une part à la distribution des situations des jeunes étudiés et d'autre part, à la distribution des situations des cohortes de référence. Des situations qui auraient été qualifiées de déclassement à partir des situations des jeunes débutant dix ans plus tôt sont devenues des situations «normales» lorsque l'évaluation du déclassement est basée sur une norme intragénérationnelle. Ce constat témoigne d'un glissement dans le temps de situations atypiques vers des situations qualifiées de «normales».

Nos résultats mettent aussi en avant le fait que selon la mesure du déclassement utilisée, l'ampleur de ce phénomène diffère. Le travail de Lizé (2005) à partir de la même enquête fait le même constat, en distinguant les emplois aidés de ceux qui ne le sont pas. Quel que soit le secteur d'emploi et après trois ans de vie active, statistiquement, $33 \%$ des jeunes sont déclassés du point de vue salarial alors qu'ils sont seulement $9 \%$ en considérant leur niveau de formation. On en déduit qu'une part des jeunes occupant des emplois de qualification correspondant à leurs formations initiales sont «mal payés», témoignant ainsi d'un désajustement entre niveau de rémunération et qualification de l'emploi. Le sentiment de déclassement reflète ce désajustement puisqu'il est également plus fréquent lorsqu'il a trait à la rémunération que lorsqu'il concerne le niveau d'études (38\% contre $28 \%$ ). Le taux de déclassement subjectif dépasse toujours le taux objectif. Cet écart est d'autant plus marqué qu'il s'agit de déclassement de niveau. La définition subjective du déclassement, du fait de la formulation de la question dans l'enquête "Génération 98 », englobe sans aucun doute d'autres dimensions de l'appréciation de la qualité de l'emploi occupé. Au-delà du lien entre formation et emploi ou entre salaire et emploi, cet indicateur recouvre d'autres éléments d'insatisfaction professionnelle.

En distinguant la fonction publique du secteur privé, indépendamment du sexe, on constate que les déclassements sont un peu plus fréquents dans ce dernier secteur, mais nettement plus grands pour le déclassement objectif de niveau. Ce résultat tient en partie à l'existence de modes de recrutement différenciés entre les deux secteurs d'emploi qui laissent une marge de manœuvre plus grande aux recruteurs du secteur privé pour embaucher des jeunes plus diplômés que ne l'exigent les postes à pourvoir. $\mathrm{Ce}$ résultat relève également de la structure des catégories socioprofessionnelles: les emplois pouvant 
Tableau 3 : Les indicateurs de déclassement à l'embauche (en \%)

\begin{tabular}{|l|c|c|c|c|c|}
\hline \multicolumn{1}{|c|}{ Indicateurs } & Ensemble & Hommes & Femmes & Fonction publique & Privé \\
\hline Déclassement subjectif de niveau & $27,6^{(*)}$ & 28,2 & 26,9 & $25,1^{(*)}$ & 27,5 \\
\hline Déclassement objectif de niveau & 9,1 & 9,9 & 8,3 & 4,4 & 10,6 \\
\hline Déclassement subjectif de salaire & 38,3 & 37,5 & 39,2 & 36,6 & 38,8 \\
\hline Déclassement objectif de salaire & 32,9 & 26,7 & 39,9 & 31,7 & 31,6 \\
\hline
\end{tabular}

Source: enquête «Génération 98 », première interrogation, CÉREQ.

Champ: ensemble des jeunes sortis en 1998 de formation initiale en emploi en 2001.

${ }^{(*)}$ Note de lecture : 27,6\% des jeunes sortis du système éducatif en 1998 en emploi en 2001 se sentent déclassés du point de vue de leur niveau de formation, c'est aussi le cas de $25,1 \%$ des jeunes en emploi dans la fonction publique.

d'autant plus donner lieu à des situations de déclassement qu'ils sont peu qualifiés sont plus nombreux dans le secteur privé.

Par ailleurs, le statut de fonctionnaire par rapport aux emplois de non titulaires s'accompagne d'un déclassement moindre qu'il soit objectif ou subjectif, alors que l'emploi en CDI ne se distingue pas des autres emplois du secteur privé du point de vue des indicateurs de déclassement (voir tableau 4). De plus, du point de vue salarial, les fonctionnaires sont moins déclassés objectivement que les jeunes en CDI du secteur privé et cela s'accompagne d'un sentiment de déclassement moins marqué.

Le genre est-il une dimension constitutive du déclassement et des différences d'ampleur entre les mesures du phénomène?

En premier lieu, les écarts de déclassement entre hommes et femmes sont faibles, hormis en ce qui concerne l'indicateur objectif salarial: $40 \%$ des femmes sont déclassées selon ce critère contre $27 \%$ des hommes. Toutefois, le sentiment de déclassement salarial n'est pas plus fréquent chez les femmes que chez les hommes. Cette insatisfaction égale pour des salaires inégaux confortent les résultats existant sur les différences d'appréciation des situations d'emploi entre les deux sexes: d'une part, les femmes sont plus satisfaites que les hommes pour un même travail car elles en ont moins d'attentes (Clark, 1997), d'autre part, les jugements des femmes minimisent l'argument salarial dans l'expression de leur satisfaction professionnelle (BAudelot, et al., 2003; Dupray, 2006) et «neutralisent l'effet de leur plus mauvaise rémunération, au point que leur satisfaction est semblable à celle des hommes» (BEssière, 2003). Ceci témoigne que les pratiques de rémunération intériorisées par les (jeunes) femmes les conduisent à accepter des salaires inférieurs à ceux des hommes à situation identique, là où les hommes exprimeraient une insatisfaction. Une telle acceptation peut être lue comme un consentement à payer de la part des femmes: acceptation d'une plus faible rentabilisation de leur formation initiale en contrepartie de ne pas être au chômage ou inactive.
Pour les hommes comme pour les femmes, c'est dans le secteur privé que le déclassement objectif de niveau est le plus important (comparativement à la fonction publique): $11 \%$ des hommes du secteur privé sont déclassés et $4,7 \%$ dans la fonction publique, respectivement $10 \%$ et $4 \%$ pour les femmes (voir tableau 4). Moins déclassées objectivement dans la fonction publique que dans le secteur privé, les femmes s'y estiment aussi moins déclassées. A contrario, les hommes se sentent plus déclassés dans la fonction publique que leurs homologues du secteur privé, bien qu'ils y soient «objectivement» moins déclassés, et ce même lorsqu'ils sont fonctionnaires.

Du point de vue du salaire, les femmes sont moins déclassées dans la fonction publique que dans le secteur privé et leur ressenti est aussi moindre. Ce résultat est encore plus net pour les fonctionnaires comparées aux femmes en CDI privé. Pour les hommes, le déclassement salarial dans la fonction publique est plus important que dans le secteur privé pour les deux critères, objectif et subjectif.

De plus, au sein de la fonction publique et toujours au regard du salaire, les femmes sont «objectivement» plus déclassées que les hommes et pourtant leur ressenti est moindre. En parallèle, la situation des femmes dans le secteur privé montre un déclassement objectif supérieur à celui des hommes, mais dans ce secteur, il va de pair avec un sentiment de déclassement plus important.

Les constats sur le déclassement salarial laissent penser à un attrait des femmes pour la fonction publique qu'on pourrait lire au travers de leur moindre sentiment de déclassement pour des situations objectives moins favorables que celles des hommes. Ils vont de pair avec la situation au regard du déclassement de niveau: les hommes ont davantage le sentiment d'être déclassés dans la fonction publique que dans le secteur privé alors qu'ils le sont moins. Ce qui les place dans une posture différente de celle des femmes à l'égard de la fonction publique. 
Tableau 4 : Déclassement selon le secteur et le genre

\begin{tabular}{|l|l|l|l|l|}
\hline \multicolumn{1}{|c|}{ Indicateurs } & \multicolumn{2}{|c|}{$\begin{array}{c}\text { Secteur privé } \\
\text { (CDI) }\end{array}$} & \multicolumn{2}{|c|}{$\begin{array}{c}\text { Fonction publique } \\
\text { (fonctionnaires) }\end{array}$} \\
\hline & Hommes & Femmes & Hommes & Femmes \\
\hline $\begin{array}{l}\text { Déclassement } \\
\text { subjectif de } \\
\text { niveau }\end{array}$ & $\begin{array}{l}27,8^{(*)} \\
\left(26,8^{(*)}\right)\end{array}$ & $\begin{array}{l}27,8 \\
(26,7)\end{array}$ & $\begin{array}{l}30,2^{(*)} \\
\left(28,2^{(*)}\right)\end{array}$ & $\begin{array}{l}24,6 \\
(20,8)\end{array}$ \\
\hline $\begin{array}{l}\text { Déclassement } \\
\text { objectif de } \\
\text { niveau }\end{array}$ & $\begin{array}{l}10,9 \\
(10,5)\end{array}$ & $\begin{array}{l}10,1 \\
(9,2)\end{array}$ & $\begin{array}{l}4,7 \\
(4,6)\end{array}$ & $\begin{array}{l}4,0 \\
(3,3)\end{array}$ \\
\hline $\begin{array}{l}\text { Déclassement } \\
\text { subjectif de } \\
\text { salaire }\end{array}$ & 37,2 & 40,7 & 38,1 & $\begin{array}{l}37,6 \\
(27,6)\end{array}$ \\
\hline $\begin{array}{l}\text { Déclassement } \\
\text { objectif de } \\
\text { salaire }\end{array}$ & $\begin{array}{l}25,9 \\
(25,5)\end{array}$ & $\begin{array}{l}(39,9) \\
(33,9)\end{array}$ & $\begin{array}{l}30,4 \\
(36,1)\end{array}$ & $\begin{array}{l}37,8 \\
(19,3)\end{array}$ \\
\hline
\end{tabular}

Source: enquête «Génération 98 », première interrogation, CÉREQ.

Champ: ensemble des jeunes sortis en 1998 de formation initiale en emploi en 2001

${ }^{(*)}$ Lecture: 27,8\% des hommes en emploi dans le secteur privé sont déclassés subjectivement du point de vue de leur niveau de formation et $26,8 \%$ des hommes en CDI dans ce secteur se sentent déclassés de ce point de vue. Dans la fonction publique, $30,2 \%$ des hommes fonctionnaires sont déclassés subjectivement du point de vue de leur niveau de formation et $28,2 \%$ sont dans ce cas pour ceux qui sont fonctionnaires.

Interpréter le décalage entre déclassement objectif et subjectif comme un consentement à payer suppose de raisonner à déclassement objectif donné et donc de faire des analyses toutes choses égales par ailleurs. Plus précisément, à même situation objective de déclassement, le fait d'avoir un ressenti moindre revient à un consentement à payer plus grand. Ainsi, si le fait d'être une femme diminue la probabilité de se sentir déclassée à situation objective donnée, on en déduira un consentement à payer plus fort des femmes, relativement aux hommes. Au-delà des constats statistiques, des analyses de corrélation décrivant le déclassement sous ses différentes formes, vont nous permettre d'avancer dans la lecture des déclassements en termes de consentements à payer. On cherche à mettre en évidence que ces consentements à payer sont liés à la fois au sexe de l'individu, à son secteur d'activité et à son statut d'emploi. On s'attend à ce que les femmes, les salariés de la fonction publique, et parmi eux, les fonctionnaires aient un consentement à payer plus fort que les autres. Le consentement à payer est-il d'autant plus marqué que la contrepartie du déclassement est la détention d'un «emploi à vie»? Autrement dit, le ressenti des femmes déclassées s'exprimerait-il moins encore lorsqu'elles sont fonctionnaires? L'accès au statut de fonctionnaire revient à la disparition du risque de chômage ou d'inactivité: le consentement à payer serait donc dans ce cas le plus grand car il annule définitivement le risque de non-emploi.

\section{Le déclassement vu comme un consentement à payer}

L'objet de cette section est d'analyser les liens entre les caractéristiques individuelles et le consentement à payer, ce dernier étant défini comme un décalage entre situation objective et situation subjective, et plus précisément comme le fait pour un individu objectivement déclassé, de ne pas le ressentir. Ainsi, à déclassement objectif contrôlé, on étudie le lien entre certaines caractéristiques individuelles et le sentiment de déclassement.

En particulier, on cherche à savoir comment le sexe, le secteur d'activité et le statut de l'emploi sont corrélés avec le sentiment de déclassement au moment de l'embauche de l'emploi occupé trois années après la sortie du système éducatif(3) pour une situation objective donnée. Il est à souligner que l'effet du déclassement objectif sur le déclassement subjectif ne nous intéresse pas en lui-même, il accroît logiquement le ressenti de déclassement, qu'il soit mesuré en termes de niveau de formation ou de salaire.

Les résultats statistiques précédents montrent que les femmes sont plus fréquemment en situation de déclassement objectif, alors qu'elles l'expriment moins souvent que les hommes. L'analyse toute chose égale par ailleurs (voir tableaux 6 et 6 bis en annexe), en particulier à secteur et déclassement objectif donnés, atteste que le ressenti est toujours moindre pour les femmes. De plus, ce ressenti (de niveau comme de salaire) est plus faible dans la fonction publique que dans le secteur privé. Pour autant, ce constat n'est pas le même selon le sexe. Plus précisément, si les femmes en emploi dans la fonction publique ont un ressenti moindre que leurs homologues du secteur privé, il n'en va pas de même pour les hommes puisque ce sont ceux salariés du secteur privé qui expriment moins souvent un sentiment de déclassement. Ainsi, le lien entre déclassement subjectif et sexe prévaut sur celui entre déclassement subjectif et secteur d'emploi.

$\mathrm{Si}$, globalement, travailler dans la fonction publique correspond à un moindre ressenti de déclassement, la stabilité de l'emploi offerte dans ce secteur accentue-t-elle ce lien?

(3) Des modèles Probit permettent d'estimer l'effet de ces facteurs sur la probabilité de se sentir déclassé. Les individus déclassés étant nécessairement en emploi, il convient de contrôler le biais de sélection de cette population en introduisant un ratio de Mill tiré de l'équation de participation au marché du travail préalablement estimée. Les résultats de ce contrôle tendent à montrer que les jeunes qui ne sont pas en emploi seraient plus déclassés que les autres, confirmant l'idée d'un arbitrage déclassement/non emploi. 
Parmi les jeunes en emploi dans la fonction publique trois ans après la fin de leurs études (4), le lien entre déclassement objectif et subjectif reste celui logique (voir tableaux 7 et 7 bis en annexe), tout comme, de manière attendu, les hommes en emploi dans ce secteur, s'estiment plus souvent déclassés que les femmes, du point de vue de leur niveau de formation comme de leur rémunération. En outre, les fonctionnaires plutôt que les non titulaires de ce statut, toutes choses étant égales par ailleurs, ont un moindre sentiment de déclassement quel qu'il soit. La stabilité de l'emploi apparaît comme un facteur minimisant le ressenti de déclassement, et ce d'autant plus pour les femmes. En effet, les femmes fonctionnaires se sentent toujours moins déclassées que toutes les autres catégories de salariés de la fonction publique. Plus précisément, lorsque le déclassement est appréhendé du point de vue des niveaux de formation, l'effet du statut l'emporte sur celui du sexe. Pour les femmes comme pour les hommes, c'est la stabilité de l'emploi qui correspond à un ressenti relativement plus faible et non pas seulement le fait d'être en emploi de la fonction publique. Lorsque la dimension salariale du déclassement est étudiée, c'est à nouveau l'effet du sexe sur le ressenti qui l'emporte, plutôt que celui du statut sur ce même ressenti.

Nous avons proposé de lire comme un consentement à payer, l'absence de sentiment de déclassement alors que celui-ci existe objectivement. Ainsi, la probabilité de ne pas se sentir déclassé sachant que les individus le sont objectivement mesure leur consentement à payer. Étudier le lien entre certaines caractéristiques individuelles et cette probabilité revient à étudier comment ces caractéristiques individuelles influencent le consentement à payer.

Ainsi, ceux sont les femmes en emploi dans la fonction publique qui ont le consentement à payer le plus important (voir tableau 5). En effet, leur probabilité de ne pas se sentir déclassées de niveau alors qu'elles le sont objectivement est de 0,534 (respectivement de 0,592 en termes salarial). Toutes choses étant égales par ailleurs (en particulier le secteur d'activité), les femmes ont un consentement à payer plus fort que celui des hommes de 4,8\% (respectivement $3,1 \%$ du point de vue du salaire). Nous avons souligné plus haut que l'effet sexe dominait l'effet secteur, ce résultat se traduit ici par le fait que la variation du consentement à payer liée au sexe est plus importante que celle liée au secteur d'emploi: travailler dans la fonction publique conduit à un consentement à payer additionnel de 1,7\% (respec-

(4) L'équation de sélection liée au fait d'être en emploi dans la fonction publique montre que, classiquement, le sexe est un facteur déterminant: toutes choses égales par ailleurs, en particulier à niveau de formation et spécialité donnés, en début de vie active, les hommes s'orientent moins souvent que les femmes vers la fonction publique. tivement $1,3 \%$ en terme salarial), quel que soit le sexe, relativement à travailler dans le secteur privé.

Tableau 5 : Consentement à payer selon le sexe, le secteur d'activité ou le statut d'emploi

\begin{tabular}{|l|r|r|}
\hline \multicolumn{1}{|c|}{ Caractéristiques } & $\begin{array}{c}\text { Consentement } \\
\text { à payer } \\
\text { en termes } \\
\text { de niveau de } \\
\text { formation }\end{array}$ & $\begin{array}{c}\text { 'onsentement } \\
\text { à payer } \\
\text { termes de } \\
\text { salaire }\end{array}$ \\
\hline $\begin{array}{l}\text { Femme dans la fonction } \\
\text { publique }\end{array}$ & $0,534^{(*)}$ & 0,592 \\
\hline $\begin{array}{l}\text { Homme dans la fonction } \\
\text { publique }\end{array}$ & $0,486^{*}\left(^{*}\right.$ & 0,561 \\
\hline $\begin{array}{l}\text { Femme dans le secteur } \\
\text { privé }\end{array}$ & 0,517 & 0,579 \\
\hline $\begin{array}{l}\text { Homme dans le secteur } \\
\text { privé }\end{array}$ & 0,469 & 0,548 \\
\hline Femme fonctionnaire & 0,663 & 0,709 \\
\hline Homme fonctionnaire & 0,619 & 0,653 \\
\hline $\begin{array}{l}\text { Femme } \\
\text { non-fonctionnaire }\end{array}$ & 0,596 & 0,653 \\
\hline $\begin{array}{l}\text { Homme } \\
\text { non-fonctionnaire }\end{array}$ & 0,552 & 0,587 \\
\hline
\end{tabular}

Source : enquête «Génération 98», première interrogation, CÉREQ

Champ: ensemble des jeunes sortis en 1998 de formation initiale en emploi en 2001.

${ }^{(*)}$ Note de lecture: les estimations des modèles Probit permettent de calculer le consentement à payer des hommes et des femmes selon leur secteur d'emploi. Ce consentement correspond à la probabilité de ne pas se sentir déclassé (de niveau de formation ou de salaire), sachant que l'individu est déclassée objectivement (de niveau de formation ou de salaire), toutes les autres variables étant considérées à leur valeur de référence. Précisément, une femme en emploi dans la fonction publique (de niveau II, de spécialité générale, résidant en province, dont le père comme la mère travaillaient dans le secteur privé à la fin des études et qui pour sa part n'a pas travaillé pendant sa formation initiale ni ressenti de discrimination au cours de son parcours professionnel) a 53,4\% de chance de ne pas se sentir déclassée alors qu'elle l'est effectivement (du point de vue de son niveau de formation). Les chances d'un homme dans la même situation sont de $48,6 \%$.

Parmi les salariés de la fonction publique, le différentiel de consentement à payer des femmes par rapport aux hommes est de $4,4 \%$ en termes de niveau de formation $(5,6 \%$ en termes de salaire); le différentiel de consentement à payer des fonctionnaires par rapport aux non-fonctionnaires est de $6,7 \%$ en termes de niveau comme de salaire.

En résumé, lorsqu'on considère la population des jeunes en emploi trois ans après la fin de leurs études, le différentiel de consentement à payer est plus fort pour les femmes (par rapport au consentement à payer des hommes) que pour les jeunes en emploi dans la fonction publique (par rapport à ceux en emploi dans le secteur privé). Quand la population est restreinte aux jeunes en emploi dans la fonction publique, c'est alors le statut davantage que le sexe qui correspond à un surcroît de ce consentement: l'écart de consentement à payer des fonctionnaires par rapport aux non titulaires de ce statut, dépasse celui des femmes relativement aux hommes. 


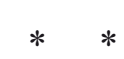

Si l'on traduit par un plus grand consentement à payer le fait d'avoir un sentiment de déclassement plus faible, à déclassement objectif donné, alors on constate, d'une part, que le consentement à payer, en terme salarial comme de niveau, des femmes en emploi dans la fonction publique est plus élevé que celui des hommes travaillant dans l'un ou l'autre des secteurs, mais aussi que celui des femmes en emploi dans le secteur privé. D'autre part, pour les femmes de la fonction publique, l'obtention d'un emploi, à vie à travers l'accès au statut de fonctionnaire, accroît le consentement à payer en termes de niveau de formation et de salaire. Ce dernier résultat, d'une prime à la stabilité conduisant à relativiser la situation objective de déclassement, est également vrai pour les hommes: les hommes (comme les femmes) fonctionnaires se sentent moins déclassés que les hommes (comme les femmes) non-fonctionnaires, à déclassement objectif donné. Ainsi, c'est le statut de fonctionnaire qui induit la plus grande variation du consentement à payer, plus que le sexe ou que le secteur d'activité et ce en terme de niveau comme de salaire.

Nous postulions dans un premier temps que, de manière générale, les femmes avaient, pour une situation de déclassement objective donnée, un sentiment de déclassement moins important que leurs homologues masculins. L'ensemble de nos résultats le confirme, allant dans le sens d'un consentement à payer plus grand pour les femmes que pour les hommes. Dans un deuxième temps, nous supposions que les femmes avaient un sentiment de déclassement plus faible lorsqu'elles travaillaient dans la fonction publique plutôt que dans le secteur privé. Cette appartenance à la fonction publique est effectivement un facteur de minimisation du ressenti plus fort pour les femmes que pour les hommes. Enfin, les résultats statistiques laissaient présager que parmi les jeunes en emploi dans la fonction publique, le statut de fonctionnaire devait diminuer le ressenti, à déclassement objectif donné. En effet, les résultats obtenus confirment que l'obtention d'un emploi à vie conduit les jeunes à relativiser le ressenti de déclassement, de niveau comme de salaire, et encore plus fortement pour les femmes que pour les hommes, toutes choses étant égales par ailleurs.

Ainsi, nos résultats valident l'idée selon laquelle les femmes exprimeraient un sentiment de déclassement moins important que leurs homologues masculins pour une même situation objective de déclassement. Par ailleurs, si travailler dans la fonction publique et de surcroît être fonctionnaire amoindrit le sentiment de déclassement pour une situation objective inchangée, c'est particulièrement le cas pour les femmes. Le consentement à payer le plus important est bien celui des femmes fonctionnaires par rapport à toutes les autres catégories de salariés, et ce en termes de niveau comme de salaire.

L'ensemble de ces résultats conforte l'idée qu'il existe un rapport spécifique des femmes à la fonction publique: y travailler leur permet de relativiser leur ressenti de déclassement. Cela n'est pas seulement lié à une tendance systématique des femmes à minimiser leur «mauvaise situation» objective, mais peut être aussi à un goût pour la sécurité de l'emploi, à la chance considérée d'être en emploi plutôt qu'au chômage, aux profils d'emploi que la fonction publique offre, aux conditions de travail, à une moindre discrimination... Si ces derniers éléments conduisent les femmes, seulement trois années après leur sortie de formation initiale, à relativiser les décalages existant, le temps passé sur le marché du travail pourrait les amener à révéler une plus grande insatisfaction au regard de leur situation objective. Aussi, il serait intéressant de s'interroger sur les évolutions comparées des consentements à payer entre hommes et femmes, entre salariés du public et salariés du privé, après plusieurs années passées sur le marché du travail. 


\section{Bibliographie}

AfFichard J. (1981), «Quels emplois après l'école : la valeur des titres scolaires depuis 1973 », Économie et Statistique, $\mathrm{n}^{\circ} 134$, pp 7-26.

Audier F. (2000), «La transmission du statut dans la fonction publique », Économie et Statistique, n $337-338$.

Bardasi E., Monfardini C. (1997), "The choice of the working sector in Italy: A trivariate probit analysis", IZA Working Paper.

Baudelot C., M. Gollac, C. Bessière, I. Coutant, O. Godechot, D. Serre, F. Viguier (2003), «Travailler pour être heureux? Le bonheur et le travail en France», Paris, Fayard.

BEssière C. (2003), «Les hommes sont-ils plus heureux au travail que les femmes?», Ecoflash, n 182.

Cahuzac E., Di Paola V. (2005), “Overeducation and Wage Downgrading: Conditions of a Spatial Differentiation", Communication aux Journées de micro-économie appliquée, Hammamet, Tunisie, juin.

CÉReQ (2002), «Quand l'école est finie... Premiers pas dans la vie active de la Génération 98», (sous la direction de Méhaut P.), (coord. Lopez A.), D. Epiphane, CEREQ.

Clark A. E. (1997), "Job satisfaction and gender: why are women so happy at work? », Labour Economics, Vol. 4, pp. 341-372.

Di Paola V., Moullet S. (2003), «L'emploi dans le secteur public et l'insertion des jeunes », Économie et Statistique, $\mathrm{n}^{\circ} 369-370$.

Di Paola V., Moullet S., Vero J. (2005) «Le déclassement dans les fonctions publiques d'État et territoriale, une analyse comparative», », in Giret, Lopez, Rose (sous la direction de), Des formations pour quels emplois? Collection «Recherches», Paris, La Découverte.

Dupray A. (2006), «L'impact du salaire sur la satisfaction dans l'emploi des femmes et des hommes selon l'univers professionnel», Communication au colloque Travail-Emploi - Formation, quelle égalité entre les hommes et les femmes? 24-25 novembre, Lille.

Fourgeot G., Gautié J. (1997), «Insertion professionnelle des jeunes et processus de déclassement», Économie et Statistique, n 304-305, pp.53-74.
Fougère D., Pouget J. (2003), «Les déterminants économiques des entrées dans la fonction publique», Économie et Statistique, $\mathrm{n}^{\circ}$ 369-370.

Garner H., Méda D., Senik C. (2005), «Conciliation entre vie professionnelle et vie familiale, les leçons des enquêtes auprès des ménages », Travail et Emploi, $\mathrm{n}^{\circ} 102$, avril-juin.

Giret J.-F. (2005), «Quand les jeunes s'estiment déclassés », in Giret, Lopez, Rose (sous la direction de), Des formations pour quels emplois? Collection «Recherches », Paris, La Découverte.

Giret J.-F., Nauze-Fichet E., Tomasini M. (2006), «Le déclassement des jeunes sur le marché du travail», in Données sociales, pp. 307-314.

Godechot O., Gurgand M. (2000), «Quand les salariés jugent leur salaire», Économie et Statistique, n ${ }^{\circ} 331$, pp. 3-24.

Goux D., Maurin E. (1993), «La sécurité de 1'emploi, une priorité croissante pour les diplômés », Économie et Statistique, $\mathrm{n}^{\circ} 261$, janvier, INSEE.

Lizé L. (2005), «Facettes du déclassement, quel rôle pour les politiques de l'emploi?», Travail et Emploi, n 107, juillet-septembre.

Mc CoRmick B. (1990), "A theory of signalling during job search, employment efficiency, and stigmatised job", Review of Economics Studies, 57, 299, 313.

Nauze-Fichet E., Tomasini M. (2001), «Diplôme et insertion sur le marché du travail: approches socioprofessionnelle et salariale du déclassement», Économie et Statistique, $\mathrm{n}^{\circ} 304$.

Nauze-Fichet E., Tomasini M. (2005), «Les jeunes en situation de déclassement sur le marché du travail: diversité des approches, diversité des éclairages » in Des formations pour quels emplois ? Giret-Lopez et Rose eds, La Découverte, colection REcherche, pp. 265-278.

ODENA S. (2005), «Les modes de garde de la petite enfance: facteurs de reproduction sociale et sexuée», thèse de doctorat en sociologie, université d'Aix-Marseille I.

SEN A.K. (1992), Inequality Reexamined, Oxford University Press. Traduction française Repenser l'inégalité, Paris, Le Seuil, 2000. 


\section{Annexes}

Nomenclature des niveaux de formation de l'éducation nationale

Niveau VI: sortie avant la troisième.

Niveau Vbis: sorties de troisième et de second cycle court avant l'année terminale.

Niveau V: sorties de l'année terminale de second cycle court professionnel et abandon de la scolarité du second cycle long avant la terminale.

Niveau IV : sorties des classes terminales du second cycle long (niveau IV secondaire) et abandon des scolarisations postbaccalauréat avant d'atteindre le niveau III (niveau IV supérieur).

Niveau III : sorties avec un diplôme de niveau bac +2 , DUT, DEUG, BTS, diplôme paramédical.

Niveau I et II : sorties avec un diplôme de deuxième ou de troisième cycle universitaire ou un diplôme de grande école.

Tableau 6: modèle Probit (1) - Estimation des probabilités de déclassement subjectif, pour la population en emploi en mars 2001

\begin{tabular}{|c|c|c|c|c|}
\hline & \multicolumn{2}{|c|}{ Déclassement de niveau } & \multicolumn{2}{|c|}{ Déclassement de salaire } \\
\hline & $\begin{array}{l}\mathrm{N}=46237 \\
\text { Wald chi2 }(22)=2109 \\
\mathrm{LnL}=-47835\end{array}$ & Prob $>$ chi $2=0.000$ & $\begin{array}{l}\mathrm{N}=46237 \\
\text { Wald chi2 }(22)=1942 \\
\mathrm{LnL}=-51858\end{array}$ & Prob $>$ chi $2=0.000$ \\
\hline Se sentir déclassé & Coef. & $\mathbf{P}>|\mathbf{z}|$ & Coef. & $\mathbf{P}>|\mathbf{z}|$ \\
\hline $\begin{array}{l}\text { Discrimination } \\
\text { ressentie }\end{array}$ & .33 & 0.000 & .25 & 0.000 \\
\hline $\begin{array}{l}\text { Travailler pendant } \\
\text { les études }\end{array}$ & .04 & 0.051 & .04 & 0.077 \\
\hline $\begin{array}{l}\text { Habiter en Ile de } \\
\text { France }\end{array}$ & .05 & 0.001 & .02 & 0.130 \\
\hline \multicolumn{5}{|l|}{ Spécialité de } \\
\hline $\begin{array}{l}\text { industrielle } \\
\text { tertiaire } \\
\text { générale }\end{array}$ & $\begin{array}{l}.13 \\
.00\end{array}$ & $\begin{array}{l}0.000 \\
0.873\end{array}$ & $\begin{array}{l}-.00 \\
-.05\end{array}$ & $\begin{array}{l}0.918 \\
0.001\end{array}$ \\
\hline \multicolumn{5}{|l|}{ Niveau de formation } \\
\hline $\begin{array}{l}\text { Niveau I } \\
\text { Niveau III } \\
\text { Niveau IV } \\
\text { Niveau IV } \\
\text { supérieur }\end{array}$ & $\begin{array}{l}-.41 \\
-.23 \\
-.24 \\
-.03\end{array}$ & $\begin{array}{l}0.000 \\
0.000 \\
0.000 \\
0.243\end{array}$ & $\begin{array}{l}-.11 \\
.18 \\
-.01 \\
.09\end{array}$ & $\begin{array}{l}0.000 \\
0.000 \\
0.639 \\
0.001\end{array}$ \\
\hline Niveau V & -.26 & 0.000 & .01 & 0.709 \\
\hline Niveau Vbis & -.38 & 0.000 & .12 & 0.008 \\
\hline Niveau VI & -.44 & 0.000 & .06 & 0.331 \\
\hline Niveau II & \multicolumn{2}{|c|}{ Ref } & \multicolumn{2}{|c|}{ Ref. } \\
\hline $\begin{array}{l}\text { Nbre d'épisodes de } \\
\text { chômage }\end{array}$ & .07 & 0.000 & .02 & 0.007 \\
\hline $\begin{array}{l}\text { Nbre d'épisodes } \\
\text { d'emploi }\end{array}$ & -.10 & 0.000 & -.01 & 0.177 \\
\hline $\begin{array}{l}\text { Nbre de mois en } \\
\text { emploi }\end{array}$ & -.00 & 0.825 & .00 & 0.000 \\
\hline \multicolumn{5}{|l|}{ Situation du père } \\
\hline $\begin{array}{l}\text { Père emploi dans } \\
\text { secteur public }\end{array}$ & .03 & 0.076 & .01 & 0.458 \\
\hline $\begin{array}{l}\text { Autre situation du } \\
\text { père }\end{array}$ & .05 & 0.007 & .04 & 0.006 \\
\hline $\begin{array}{l}\text { Père emploi dans } \\
\text { secteur privé }\end{array}$ & \multicolumn{2}{|c|}{ Ref. } & \multicolumn{2}{|c|}{ Ref. } \\
\hline \multicolumn{5}{|l|}{ Situation de la mère } \\
\hline $\begin{array}{l}\text { Mère emploi dans } \\
\text { secteur public }\end{array}$ & .02 & 0.242 & .05 & 0.003 \\
\hline $\begin{array}{l}\text { Autre situation de } \\
\text { la mère }\end{array}$ & .02 & 0.210 & -.00 & 0.759 \\
\hline $\begin{array}{l}\text { Mère emploi dans } \\
\text { secteur privé }\end{array}$ & \multicolumn{2}{|c|}{ Ref. } & \multicolumn{2}{|c|}{ Ref. } \\
\hline Homme & .12 & 0.000 & .08 & 0.000 \\
\hline $\begin{array}{l}\text { être en emploi dans } \\
\text { la FP* }\end{array}$ & -.04 & 0.006 & -.03 & 0.022 \\
\hline $\begin{array}{l}\text { être déclassé } \\
\text { objectivement }\end{array}$ & .68 & 0.000 & .54 & 0.000 \\
\hline IMR & .33 & 0.001 & .38 & 0.000 \\
\hline Constante & -.58 & 0.000 & -.83 & 0.000 \\
\hline
\end{tabular}


FEMMES ET FONCTION PUBLIQUE : UN RISQUE CALCULÉ DE DÉCLASSEMENT

\begin{tabular}{|c|c|c|}
\hline \multirow{2}{*}{$\begin{array}{l}\text { Equation de } \\
\text { sélection }\end{array}$} & \multicolumn{2}{|c|}{ Probabilité d'être en emploi à la date d'enquête } \\
\hline & Coef. & $\mathbf{P}>|\mathbf{z}|$ \\
\hline Homme & .29 & 0.000 \\
\hline Avoir un enfant & -.49 & 0.000 \\
\hline Etre en couple & .34 & 0.000 \\
\hline \multicolumn{3}{|l|}{ Situation du père } \\
\hline $\begin{array}{l}\text { Père emploi ds } \\
\text { secteur public }\end{array}$ & -.04 & 0.036 \\
\hline $\begin{array}{l}\text { Autre situation } \\
\text { du père }\end{array}$ & -.12 & 0.000 \\
\hline $\begin{array}{l}\text { Père emploi ds } \\
\text { secteur privé }\end{array}$ & \multicolumn{2}{|c|}{ Ref. } \\
\hline \multicolumn{3}{|l|}{$\begin{array}{l}\text { Situation de la } \\
\text { mère }\end{array}$} \\
\hline $\begin{array}{l}\text { Mère emploi ds } \\
\text { secteur public }\end{array}$ & -.07 & 0.000 \\
\hline $\begin{array}{l}\text { Autre situation } \\
\text { de la mère }\end{array}$ & -.09 & 0.000 \\
\hline $\begin{array}{l}\text { Mère emploi ds } \\
\text { secteur privé }\end{array}$ & \multicolumn{2}{|c|}{ Ref. } \\
\hline \multicolumn{3}{|l|}{ Niveau de } \\
\hline \multicolumn{3}{|l|}{ formation } \\
\hline Niveau I & .22 & 0.000 \\
\hline Niveau III & .08 & 0.000 \\
\hline Niveau IV & -.32 & 0.000 \\
\hline Niveau IV & -.31 & 0.000 \\
\hline supérieur & & \\
\hline Niveau $\mathrm{V}$ & -.55 & 0.000 \\
\hline Niveau Vbis & -.90 & 0.000 \\
\hline Niveau VI & -.81 & 0.000 \\
\hline Niveau II & \multicolumn{2}{|c|}{ Ref. } \\
\hline \multirow{2}{*}{\multicolumn{3}{|c|}{$\begin{array}{l}\text { Spécialité de } \\
\text { formation }\end{array}$}} \\
\hline & & \\
\hline industrielle & -.39 & 0.000 \\
\hline tertiaire & .07 & 0.000 \\
\hline générale & \multicolumn{2}{|c|}{ Ref. } \\
\hline Père né en Afrique & -.19 & 0.000 \\
\hline Père né à & -.01 & 0.607 \\
\hline \multicolumn{3}{|l|}{ l'étranger hors } \\
\hline \multicolumn{3}{|l|}{ Afrique } \\
\hline Père né en France & \multicolumn{2}{|c|}{ Ref. } \\
\hline Constante & 1.26 & 0.000 \\
\hline
\end{tabular}

Tableau 6bis : Estimation du modèle 1 avec introduction de variables croisant le sexe et le secteur d'emploi

\begin{tabular}{|c|c|c|c|c|}
\hline & \multicolumn{2}{|c|}{ Déclassement de niveau } & \multicolumn{2}{|c|}{ Déclassement de salaire } \\
\hline & \multicolumn{2}{|c|}{$\begin{array}{c}\mathrm{N}=46237 \\
\text { Wald chi } 2(23)=2126 \\
\text { Prob }>\text { chi } 2=0.000 \\
\text { LnL }=-47826\end{array}$} & \multicolumn{2}{|c|}{$\begin{array}{c}\mathrm{N}=46237 \\
\text { Wald chi2 }(23)=1952 \\
\text { Prob }>\text { chi } 2=0.000 \\
\text { LnL }=-51853\end{array}$} \\
\hline Déclassement subjectif & Coef. & $\mathbf{P}>|\mathbf{z}|$ & Coef. & $\mathbf{P}>|\mathbf{z}|$ \\
\hline Homme fonction publique & .22 & 0.000 & .15 & 0.000 \\
\hline Homme privé & .19 & 0.000 & .13 & 0.000 \\
\hline Femme fonction publique & \multicolumn{2}{|c|}{ Réf } & \multirow{2}{*}{\multicolumn{2}{|c|}{ Réf. }} \\
\hline Femme privé & .10 & 0.000 & & \\
\hline
\end{tabular}

(Toutes les autres variables étant contrôlées, même modélisation que le modèle 1 - tableau 5 - estimation disponible à la demande aux auteurs). 
Tableau 7 : modèle 2 Probit - Estimation des probabilités de déclassement subjectif pour la population en emploi dans la fonction publique en mars 2001

\begin{tabular}{|c|c|c|c|c|}
\hline \multirow[b]{3}{*}{ Se sentir déclassé } & \multirow{2}{*}{\multicolumn{2}{|c|}{$\begin{array}{c}\text { Déclassement de niveau } \\
\mathrm{N}=11066 \\
\text { Wald chi } 2(25)=856 \\
\text { Prob }>\text { chi } 2=0.000 \\
\text { LnL }=-31002\end{array}$}} & \multirow{2}{*}{\multicolumn{2}{|c|}{$\begin{array}{c}\text { Déclassement de salaire } \\
\mathrm{N}=11066 \\
\text { Wald chi2 }(25)=778 \\
\text { Prob }>\text { chi } 2=0.000 \\
\text { LnL }=-32079\end{array}$}} \\
\hline & & & & \\
\hline & Coef. & $\mathbf{P}>|\mathbf{z}|$ & Coef. & $\mathbf{P}>|\mathbf{z}|$ \\
\hline $\begin{array}{l}\text { Secteur de la FP } \\
\text { FP territoriale } \\
\text { FP hospitalière } \\
\text { Autre FP } \\
\text { Education nat. }\end{array}$ & $\begin{array}{l}.11 \\
-.40 \\
.06\end{array}$ & $\begin{array}{l}0.014 \\
0.000 \\
0.069\end{array}$ & $\begin{array}{l}.13 \\
-.07 \\
-.03\end{array}$ & $\begin{array}{l}0.003 \\
0.108 \\
0.443\end{array}$ \\
\hline Discrimination ressentie & .37 & 0.000 & .23 & 0.000 \\
\hline Travailler pendant les études & .11 & 0.003 & .01 & 0.707 \\
\hline Habiter en Ile de France & .08 & 0.025 & .15 & 0.000 \\
\hline $\begin{array}{l}\text { Spécialité de formation } \\
\text { industrielle } \\
\text { tertiaire } \\
\text { générale } \\
\end{array}$ & $\begin{array}{l}.25 \\
.08\end{array}$ & $\begin{array}{l}0.000 \\
0.340\end{array}$ & $\begin{array}{l}.09 \\
-.07\end{array}$ & $\begin{array}{l}0.010 \\
0.394\end{array}$ \\
\hline $\begin{array}{l}\text { Niveau de formation } \\
\text { Niveau I } \\
\text { Niveau III } \\
\text { Niveau IV } \\
\text { Niveau IV supérieur } \\
\text { Niveau V } \\
\text { Niveau Vbis } \\
\text { Niveau VI } \\
\text { Niveau II } \\
\end{array}$ & $\begin{array}{l}-.52 \\
-.29 \\
-.37 \\
-.13 \\
-.41 \\
-.42 \\
-.60\end{array}$ & $\begin{array}{l}0.000 \\
0.000 \\
0.002 \\
0.130 \\
0.007 \\
0.032 \\
0.041\end{array}$ & $\begin{array}{l}.36 \\
.31 \\
-.00 \\
.20 \\
-.03 \\
-.17 \\
-.27\end{array}$ & $\begin{array}{l}0.000 \\
0.000 \\
0.979 \\
0.034 \\
0.860 \\
0.438 \\
0.387\end{array}$ \\
\hline $\begin{array}{l}\text { Nbre d'épisodes de chômage } \\
\text { Nbre d'épisodes d'emploi } \\
\text { Nbre de mois en emploi }\end{array}$ & $\begin{array}{l}.12 \\
-.18 \\
.00\end{array}$ & $\begin{array}{l}0.000 \\
0.000 \\
0.021\end{array}$ & $\begin{array}{l}.04 \\
-.03 \\
.01\end{array}$ & $\begin{array}{l}0.036 \\
0.038 \\
0.000\end{array}$ \\
\hline \multicolumn{5}{|l|}{ Situation du père } \\
\hline $\begin{array}{l}\text { Père emploi ds secteur } \\
\text { public } \\
\text { Autre situation du père } \\
\text { Père emploi ds secteur privé }\end{array}$ & $\begin{array}{l}.02 \\
.01\end{array}$ & $\begin{array}{l}0.670 \\
0.793\end{array}$ & $\begin{array}{l}.02 \\
.06\end{array}$ & $\begin{array}{l}0.755 \\
0.150\end{array}$ \\
\hline \multicolumn{5}{|l|}{\begin{tabular}{l|l} 
Situation de la mère \\
\end{tabular}} \\
\hline $\begin{array}{l}\text { Mère emploi ds secteur } \\
\text { public } \\
\text { Autre situation de la mère } \\
\text { Mère emploi ds secteur } \\
\text { privé }\end{array}$ & $\begin{array}{l}.09 \\
.07\end{array}$ & $\begin{array}{l}0.025 \\
0.025\end{array}$ & $\begin{array}{l}.08 \\
.06\end{array}$ & $\begin{array}{l}0.070 \\
0.047\end{array}$ \\
\hline $\begin{array}{l}\text { Homme } \\
\text { être fonctionnaire } \\
\text { être déclassé objectivement. } \\
\text { IMR } \\
\text { Constante }\end{array}$ & $\begin{array}{l}.11 \\
-.17 \\
.60 \\
-.15 \\
-.70\end{array}$ & $\begin{array}{l}0.003 \\
0.000 \\
0.000 \\
0.523 \\
0.003\end{array}$ & $\begin{array}{l}.15 \\
-.18 \\
.57 \\
.05 \\
-1.15\end{array}$ & $\begin{array}{l}0.000 \\
0.000 \\
0.000 \\
0.821 \\
0.000\end{array}$ \\
\hline
\end{tabular}


FEMMES ET FONCTION PUBLIQUE : UN RISQUE CALCULÉ DE DÉCLASSEMENT

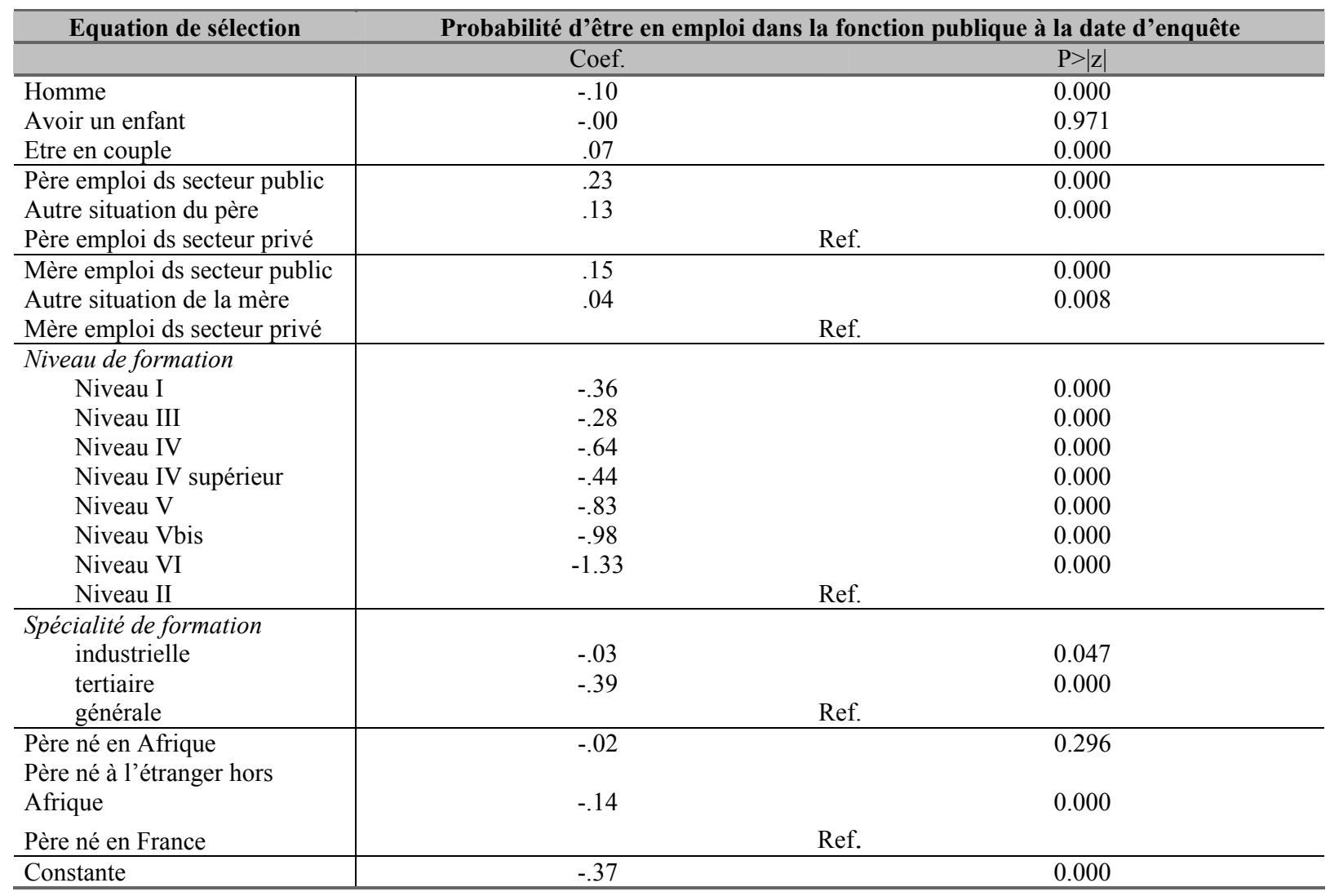

Tableau 7 bis : Estimation du modèle 2 avec introduction de variables croisant le sexe et le statut (fonctionnaire ou non)

\begin{tabular}{|c|c|c|c|c|}
\hline & \multicolumn{2}{|c|}{ Déclassement de niveau } & \multicolumn{2}{|c|}{ Déclassement de salaire } \\
\hline & \multicolumn{2}{|c|}{$\begin{array}{c}\mathrm{N}=11066 \\
\text { Wald } \operatorname{chi} 2(26)=864 \\
\text { Prob }>\text { chi } 2=0.000 \\
\mathrm{LnL}=-31000\end{array}$} & \multicolumn{2}{|c|}{$\begin{array}{c}\mathrm{N}=11066 \\
\text { Wald chi2 }(26)=787 \\
\text { Prob }>\text { chi2 }=0.000 \\
\mathrm{LnL}=-32074\end{array}$} \\
\hline Déclassement subjectif & Coef. & $\mathbf{P}>|\mathbf{z}|$ & Coef. & $\mathbf{P}>|\mathbf{z}|$ \\
\hline Homme fonctionnaire & .19 & 0.000 & .28 & 0.000 \\
\hline Homme non fonctionnaire & .30 & 0.000 & .34 & 0.000 \\
\hline Femme fonctionnaire & & & & \\
\hline Femme non fonctionnaire & .23 & 0.000 & .26 & 0.000 \\
\hline
\end{tabular}

(Toutes les autres variables étant contrôlées, même modélisation que le modèle 2 - tableau 6 - estimation disponible à la demande aux auteurs). 